\title{
HSC/19/01
}

\section{Electricity price forecasting}

\section{Katarzyna Maciejowska ${ }^{1}$ Rafał Weron ${ }^{1}$}

${ }^{1}$ Department of Operations Research, Faculty of Computer Science and Management, Wrocław University of Science and Technology, Poland

\section{Hugo Steinhaus Center}

Wrocław University of Science and Technology Wyb. Wyspiańskiego 27, 50-370 Wrocław, Poland http://www.im.pwr.wroc.pl/ hugo/ 


\title{
Electricity Price Forecasting
}

\section{Katarzyna Maciejowska ${ }^{a}$ and Rafał Weron ${ }^{\mathrm{a} *}$}

Keywords: Forecasting; Electricity Spot Price; Day-ahead Market; Variable Selection; Regularization;

Regression; Quantile Regression

\begin{abstract}
Electricity price forecasting (EPF) is an actively developing research field, which aims at predicting the spot and forward prices in wholesale electricity markets. Since day-ahead forecasting has gained the most attention, in this article we review the modeling approaches for short-term predictions, with a particular focus on variable selection. Copyright (c) 2019 John Wiley \& Sons, Ltd.
\end{abstract}

\section{Introduction}

Electricity is a very special commodity. Despite the recent progress in battery performance, it still is largely a nonstorable good. System security requires a constant balance between production and consumption, both of which are dependent on weather conditions and the intensity of business activities. On one hand, the resulting spot prices exhibit seasonality at the daily, weekly and - depending on the geographical location - also annual levels. On the other, they are volatile compared to other commodities and tend to spike - upward due to scarcity of supply (e.g., excessive use of air conditioning during a hot spell) and downward due to abundance of generation from renewable sources in low demand periods (e.g., high winds during a holiday or at night). In some markets, the latter may lead to negative prices.

In the electricity price forecasting (EPF) literature, it is customary to talk about short-, medium- and long-term predictions. However, there are no commonly accepted definitions. Short-term horizons range from minutes to days and are mainly relevant for market operations and system stability. Since scheduling and trading decisions are made through revenue contribution models and generally are benchmarked against the spot prices, short-term EPF has gained the most attention (Chen \& Bunn 2014, Weron 2014). It is also the focus of this article.

Medium-term refers to horizons for which reliable meteorological predictions are not available. With lead times measured in weeks, months, quarters or years, medium-term EPF is used for maintenance scheduling, resource reallocation, derivatives valuation, risk management and budgeting. Finally, long-term predictions refer to everything beyond a couple of years and are used for investment planning and policy making (Ziel \& Steinert 2018).

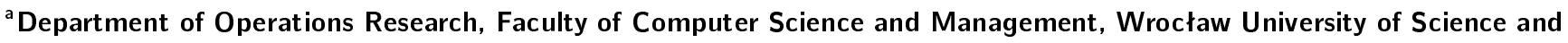
Technology, Wrocław, Poland

*Email: \{katarzyna.maciejowska, rafal.weron\}@pwr.edu.pl

Partially supported by the National Science Center (NCN, Poland) through grants 2016/21/D/HS4/00515 (to KM) and 2015/17/B/HS4/00334 (to RW).
} 


\section{Modeling frameworks for short-term predictions}

In most electricity markets, particularly European, the 'spot price' is actually a very short-term forward price determined around noon during 24 uniform price auctions - one for each hour of the next day; hence the term day-ahead price. Since they are established using the same information set, it is customary to use double indexing, i.e., $P_{d, h}$, when referring to the day-ahead price for day $d$ and hour $h$, and use a set of 24 (possibly interrelated) models, one for each hour of the day (see Ziel \& Weron, 2018, for a discussion of the uni- and multivariate structures used in EPF).

\subsection{Multiple regression}

One of the most popular techniques and a basic building block for more sophisticated electricity price (and demand) forecasting models is multiple regression. It represents a dependent (criterion, output) variable by a linear combination of several independent (predictor, explanatory) variables, also called regressors, inputs or features:

$$
P_{d, h}=\boldsymbol{\beta}_{h} \mathbf{X}_{d, h}+\varepsilon_{d, h},
$$

where $\boldsymbol{\beta}_{h}=\left[\beta_{h, 0}, \beta_{h, 1}, \ldots, \beta_{h, k}\right]$ is a $1 \times(k+1)$ vector of coefficients specific to hour $h, \mathbf{X}_{d, h}=\left[1, X_{d, h}^{1}, \ldots, X_{d, h}^{k}\right]^{\prime}$ is a $(k+1) \times 1$ vector of inputs and $\varepsilon_{d, h}$ is an error term, also specific to hour $h$. The first 'regressor' is the intercept and $\beta_{h, 0}$ can be set to zero if the data is demeaned beforehand. The remaining $k$ variables can be - and usually are - transformed before estimating $\boldsymbol{\beta}_{h}$, see Uniejewski et al. (2018) for suggestions. For instance, a commonly used benchmark, originally proposed by Misiorek et al. (2006) and later used in a number of EPF studies, takes the form an ARX (autoregressive with exogenous variables) model:

$$
\begin{aligned}
P_{d, h}= & \beta_{h, 0}+\underbrace{\beta_{h, 1} P_{d-1, h}+\beta_{h, 2} P_{d-2, h}+\beta_{h, 3} P_{d-7, h}+\beta_{h, 4} P_{d-1, \text { min }}}_{\text {past prices (autoregressive effects) }} \\
& +\underbrace{\beta_{h, 5} L_{d, h}}_{\text {load forecast }}+\underbrace{\beta_{h, 6} D_{S a t}+\beta_{h, 7} D_{\text {Sun }}+\beta_{h, 8} D_{M o n}}_{\text {weekday dummies }}+\varepsilon_{d, h},
\end{aligned}
$$

where $P_{d-1, \min }$ is the minimum of the previous day's 24 hourly prices, $L_{d, h}$ refers to the load forecast for day $d$ and hour $h$ (known on day $d-1$ ), and the three dummies $\left(D_{S a t}, D_{\text {Sun }}, D_{M o n}\right)$ model the weekly pattern, with higher prices during the weekdays and lower on the weekends (and Monday morning).

The regression model is typically estimated via ordinary least squares (OLS) and using electricity prices from the past $D$ days, i.e., $P_{1, h}, \ldots, P_{D, h}$, to predict the next day's price $P_{D+1, h}$; the calibration sample may be limited to hour $h$ or include some or all of the remaining hours. Like with the classification of forecasting horizons, there is no consensus in the literature as to the value of $D$. Many studies consider a 'year' $(D=360,364,365)$ or 'two years' $(D=728,730)$ of data, but some use as short calibration windows as 10 days, while other as long as six years. Hubicka et al. (2019) actually argue that combining predictions obtained from a model calibrated to windows of different lengths (e.g., 2-3 short- and 2-3 long-term) outperforms selecting ex-ante only one 'optimal' window length.

\subsection{Neural networks}

In the engineering stream of the EPF literature, neural networks are the most popular modeling framework (Weron 2014). A single-layer perceptron with only input and an output node is equivalent to a linear regression. By adding an intermediate layer with hidden nodes we obtain the multi-layer perceptron (MLP), with a feed-forward architecture but already able to represent non-linear phenomena, see Fig. 1 When the network has more than one hidden layer it is called a deep neural network (DNN) and when connections between nodes form directed loops to the same or other 


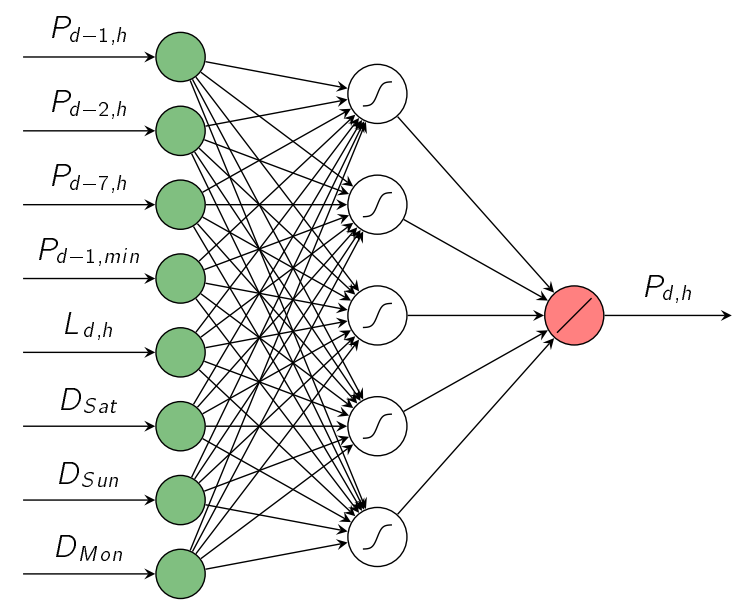

Figure 1. A multilayer perceptron with the same inputs (green nodes) and output (red node) as the regression in Eqn. (2), and one hidden layer composed of five neurons with sigmoid activation functions (white nodes). The hidden layer makes this a non-linear model; adding another hidden layer would yield a deep neural network (DNN).

layers (not a feed-forward architecture) it is referred to as a recurrent neural network (RNN); the latter is particularly useful for modeling temporal dynamic behavior. Both extensions form the backbone of deep learning and have become extremely popular in the last few years (Goodfellow et al. 2016). Training of such structures is more complex and time consuming than of regression models. The standard approach is based on gradient descent and called back-propagation through time (BPTT); architectures composed of long short-term memory (LSTM) units or their simpler version gated recurrent units (GRU) - are used to avoid the vanishing gradient problem (Schmidhuber 2015).

\subsection{Multivariate modeling}

Note, that both the regression and neural network approaches admit multivariate modeling, where one model returns a vector of 24 hourly prices. In a regression context, the model structure is composed of 24 Eqns. (1) and is jointly estimated for all $h=1,2, \ldots, 24$, e.g., via multivariate least squares, multivariate Yule-Walker equations or maximum likelihood (Lütkepohl 2005); the resulting model is called VAR (vector autoregressive). In a neural network setting, the output layer may simply consist of 24 nodes and likely require more hidden neurons to adequately represent the dependencies for all hours. Note, however, that while multivariate models usually provide a better in-sample fit, their predictive performance does not have to be better (Chatfield, 2000). This, indeed, is observed for VAR models in the extensive EPF study of Ziel \& Weron (2018).

\section{Variable selection}

No matter which framework the modeler is using, a key point in EPF is an appropriate choice of explanatory variables (Abedinia et al., 2017 Karakatsani \& Bunn 2008, Keles et al. 2016). Most studies select predictors using expert knowledge (hence the resulting structures are dubbed expert models; see Ziel, 2016), rarely a formal automated procedure for variable selection is implemented. The most common classes of inputs are reviewed in Sections 3.1 and 3.2 then automated variable selection is briefly discussed in Section 3.3 


\subsection{Seasonal components}

Two types of components are distinguished in the EPF literature: short- and long-term. The short-term seasonal component (STSC) represents the weekly pattern; the intra-day behavior is typically accounted for by the multivariate structure of the model. In the majority of EPF studies, the STSC is introduced in the form of dummy variables, either one for each day of the week or only for the less typical days, as in Eqn. (2) and Fig. 1 . Holidays - if considered at all - are treated as the 8 th day of the week or grouped with Sundays. Uniejewski et al. (2016) and Ziel \& Weron (2018) emphasize, that the importance of daily dummies depends on the hour of the day and suggest to incorporate periodic effects (e.g., daily dummies multiplied by $P_{d-1, h}$ ) in general model design.

On the other hand, the long-term seasonal component (LTSC) aims at capturing the annual or - the less regular and periodic - trend-seasonal behavior associated with macroeconomic and latent factors. There are three popular approaches in the EPF literature (Weron 2014):

- piecewise constant functions or monthly dummies,

- sinusoidal functions or their sums across different frequencies (based on Fourier decomposition),

- nonparametric smoothing techniques, like wavelets, Friedman's supersmoother, the Hodrick-Prescott filter, spline functions, empirical mode decomposition or singular spectrum analysis.

While extrapolating into the future the first two classes of LTSCs is straightforward, the latter requires additional assumptions. Nevertheless, as Nowotarski et al. (2013) show, this extra work is worthwhile. When only day-ahead predictions of the LTSC are required, a naive (persistent) forecast works reasonably well, leading to the seasonal component (SCAR, SCANN) modeling approach (Marcjasz et al. 2018).

\subsection{Stochastic variables}

Electricity prices. In the ARX model defined in Eqn. (2), the autoregressive effects are limited to the same hour yesterday, two days ago and a week ago, and a minimum of yesterday's prices. The former variable is typically regarded in the EPF literature as the most important regressor. However, as Ziel \& Weron (2018) show, an even more influential explanatory variable may be $P_{d-1,24}$, i.e., the last known price, or - more generally - yesterday's late evening prices. Other 'less intuitive' regressors include yesterday's prices for the neighboring hours (e.g., $P_{d-1,18}$ when predicting $P_{d, 17}$ ) and very recent prices of daily futures contracts with delivery on day $d$, and - what may seem even more surprising - with delivery on day $d+1$, but only when predicting the evening hours 20-24 (Steinert \& Ziel 2019). A likely explanation for the latter is that the evening hours of day $d$ are close to the early morning hours of day $d+1$, when the delivery of these futures contracts begin.

Demand. Most studies agree that the demand for electricity is one of the most important fundamental variables (Karakatsani \& Bunn, 2008, Weron 2014). Quite often it is referred to as load, although load is an ambiguous term and may mean other things. In load (and price) forecasting, the load usually means demand (in $\mathrm{kW}$ ) or consumed energy (kWh). On hourly data, the magnitude of power and energy is the same, so the authors rarely emphasize whether it is demand or energy (Hong 2014). The information on predicted for the next day and past demand levels is available for many markets and is typically published by the TSO (Transmission System Operator). However, as Uniejewski \& Weron (2018) show, past loads do not provide any additional information and, therefore, the influence of this fundamental variable can be limited to the day-ahead load forecast for a particular hour, as $L_{d, h}$ in Eqn. (2) and Fig. 1 


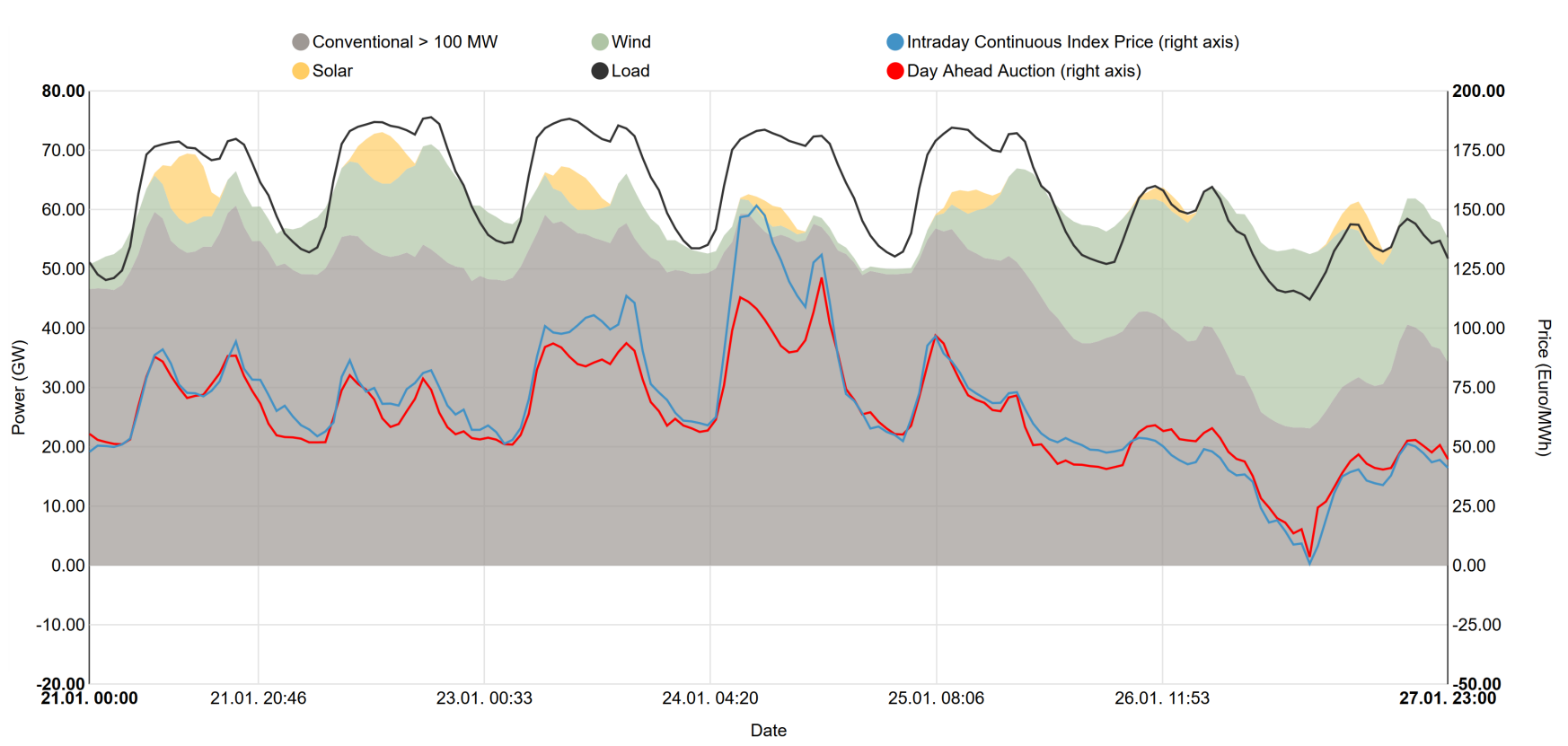

Figure 2. Main generation sources (conventional, wind, solar) and wholesale electricity prices (day-ahead, intraday) for the 4th week of 2019 in Germany. Source: https://www. energy-charts.de Fraunhofer ISE.

Generation structure. Many TSOs publish information about the actual and predicted generation structure, indicating the amount of electricity generated from different sources, e.g., nuclear, coal, gas and RES (renewable energy sources; primarily hydro, wind and solar). The intermittent RES generation has a decisive role in the price setting process (Gianfreda et al. 2016, Kiesel \& Paraschiv 2017: Uniejewski \& Weron, 2018). Since in many countries RES has a priority during the dispatch, changes in RES lead to a shift of the supply curve - a phenomenon called the merit-order effect - and hence affect the electricity prices. This is nicely illustrated in Fig. 2 for the German market. A drop in wind power generation on Thursday leads to an increase of prices, particularly in the intra-day market, while an increase of wind generation drags the prices almost to zero on Saturday-to-Sunday night. However, the literature is inconclusive about how the shifts in the supply curves impact price volatility. For instance, Rintamäki et al. (2017) report that in Denmark wind power decreases the volatility by flattening the price profile, but in Germany the effect is the opposite because wind power has a stronger impact on off-peak prices, while the in-feed of solar power decreases the volatility. It is also not clear, which is more relevant for EPF: the aggregated measure of RES or individual information on solar and wind generation.

Generation costs. Fuel and $\mathrm{CO}_{2}$ emission allowance prices have a direct impact on the generation costs. Due to the merit-order effect, the marginal generation technology changes across the day, affecting the fuel-electricity price relationship. For instance, gas prices impact mainly the peak hours, whereas coal prices influence the off-peak hours. However, these dependencies can be observed and utilized only for the medium- and long-term forecasting horizons (Maciejowska \& Weron 2016 Ziel \& Steinert, 2018). The influence of $\mathrm{CO}_{2}$ emission allowance prices is even less pronounced. Due to excess supply, their prices remained at very low levels until 2017. Last year's changes in EU regulations have resulted in a price hike, which - if sustained - may increase their impact on electricity prices in the years to come. Still, this will rather be a medium- or long-term phenomenon. 


\subsection{Automated variable selection}

The OLS estimator used to fit a regression model to data is unbiased, but can have a non-negligible variance. Especially when the regressors are correlated with each other and/or when there are many of them. The first attempts to limit the number of inputs in EPF models in a systematic way used stepwise regression (Karakatsani \& Bunn, 2008) and feature selection based on mutual information (for neural networks; Amjady \& Keynia 2009).

The concept of regularization (or shrinkage) entered the EPF literature a few years later and quickly brought a qualitative change. Recall, that it aims at lowering the variance (at the cost of some bias) by penalizing the fit if the estimated $\beta_{h, i}$ 's in Eqn. (1) are too large; effectively it shrinks them towards or even exactly to zero. In the EPF literature, Uniejewski et al. (2016) and Ziel (2016) used LASSO (least absolute shrinkage and selection operator) and elastic nets to sparsify very large $(100+)$ sets of regression coefficients. Even larger models of ca. 200 and ca. 400 regressors were considered by Ziel \& Weron (2018) and Uniejewski \& Weron (2018), respectively. In all cases, the elastic net or LASSO-implied structures significantly outperformed the autoregressive benchmarks. Interestingly, as a by-product of the selection property of these techniques, parsimonious regression structures able to outperform commonly used expert models can be identified.

Combining the ideas of machine learning and sparsity we arrive at a relatively new concept of regularizing neural network architectures (He \& Liu 2018). It has not been utilized in EPF to date. However, given the potential of deep architectures to model nonlinear time series data on one hand and the increase of computational power and advances in training algorithms on the other, this approach may see a widespread use in the near future.

\section{Beyond point forecasts}

Although over $90 \%$ of all EPF studies published to date focus on point forecasting, in the last few years both the academics and practitioners realized that there is much to be gained from computing probabilistic and ensemble forecasts. Probabilistic EPF gained popularity with the Global Energy Forecasting Competition 2014 (GEFCom2014; Hong et al. 2016), which required participants to predict 99 percentiles of next day's load, electricity price, wind or solar power (for each of the 24 hours). Since then the number of probabilistic EPF papers has increased rapidly, see Nowotarski \& Weron (2018) and Ziel \& Steinert (2018) for reviews.

There are two main approaches to computing probabilistic forecasts: the first one utilizes the point prediction $\hat{P}_{d, h}$ and the distribution $F_{\varepsilon}$ of errors associated with it, the second directly approximates the price distribution $F_{P}$. Among the error distribution-based methods, the noise, e.g., $\varepsilon_{d, h}$ in Eqn. (2), is typically assumed to have a normal or Student$t$ distribution, possibly embedded in a GARCH (generalized autoregressive conditional heteroscedasticity) component to better model time varying moments. If the noise term does not take any particular distributional form, as in the case of neutral networks, the bootstrap is commonly used to empirically approximate $F_{P}$ (see Nowotarski \& Weron 2018, for details). Another popular semi-parametric method is quantile regression (QR; Koenker 2005), which directly models the $\tau$-th quantile of the price as a function of exogenous variables:

$$
Q_{\tau}\left(P_{d, h}\right)=\boldsymbol{\beta}_{h}^{\tau} \mathbf{X}_{d, h},
$$

where $\boldsymbol{\beta}_{h}^{\tau}$ is a $1 \times(k+1)$ vector of parameters, which depends on hour $h$ and quantile $\tau$; unlike in Eqn. (1), here the intercept needs to be included among the regressors. Model parameters are estimated by minimizing the so-called pinball score. QR can be used to forecast two chosen quantiles such that $\operatorname{Prob}\left(P_{d, h} \in\left[\boldsymbol{\beta}_{h}^{\tau_{1}} \mathbf{X}_{d, h}, \boldsymbol{\beta}_{h}^{\tau_{2}} \mathbf{X}_{d, h}\right]\right)=\tau_{2}-\tau_{1}$, as in the QRA (Quantile Regression Averaging) method of Nowotarski \& Weron (2015), or to approximate the whole price distribution by computing a range of quantiles, e.g., $\tau=0.01, \ldots, 0.99$ as in Maciejowska \& Nowotarski (2016). 
Ensemble forecasts, on the other hand, have not seen application in EPF as yet. This is surprising given the popularity in the meteorological and wind power forecasting literature (Pinson 2013), as well as a plethora of optimization problems where path-dependency is crucial (e.g., power plant scheduling, energy storage, day-ahead trading). An ensemble is a collection of paths (trajectories, scenarios) from a forecasting model, typically simulated using Monte Carlo. For a large number of paths the ensemble can approximate the 24-dimensional distribution of the 24-dimensional price vector $\mathbf{P}_{\mathrm{d}}=\left(P_{d, 1}, \ldots, P_{d, 24}\right)^{\prime}$ reasonably well. For many EPF models this is the only feasible solution, since they cannot directly provide such a multidimensional distributional forecast.

\section{References}

Abedinia, O, Amjady, N \& Zareipour, H (2017), 'A new feature selection technique for load and price forecast of electrical power systems,' IEEE Transactions on Power Systems, 32(1), pp. 62-74.

Amjady, N \& Keynia, F (2009), 'Day-ahead price forecasting of electricity markets by mutual information technique and cascaded neuro-evolutionary algorithm,' IEEE Transactions on Power Systems, 24(1), pp. 306-318.

Chatfield, C (2000), Time-Series Forecasting, Chapman \& Hall/CRC, Boca Raton, Florida.

Chen, D \& Bunn, D (2014), 'The forecasting performance of a finite mixture regime-switching model for daily electricity prices,' Journal of Forecasting, 33(5), pp. 364-375.

Gianfreda, A, Parisio, L \& Pelagatti, M (2016), 'The impact of RES in the Italian day-ahead and balancing markets,' Energy Journal, 37, pp. 161-184.

Goodfellow, I, Bengio, Y \& Courville, A (2016), Deep Learning, MIT Press.

He, W \& Liu, Y (2018), 'To regularize or not: Revisiting SGD with simple algorithms and experimental studies,' Expert Systems with Applications, 112, pp. 1-14.

Hong, T (2014), 'Load, demand, energy and power,' http://blog.drhongtao.com/2014/09/load-demand-energypower.html.

Hong, T, Pinson, P, Fan, S, Zareipour, H, Troccoli, A \& Hyndman, RJ (2016), 'Probabilistic energy forecasting: Global Energy Forecasting Competition 2014 and beyond,' International Journal of Forecasting, 32(3), pp. 896-913.

Hubicka, K, Marcjasz, G \& Weron, R (2019), 'A note on averaging day-ahead electricity price forecasts across calibration windows,' IEEE Transactions on Sustainable Energy, 10(1), pp. 321-323.

Karakatsani, N \& Bunn, D (2008), 'Forecasting electricity prices: The impact of fundamentals and time-varying coefficients,' International Journal of Forecasting, 24, pp. 764-785.

Keles, D, Scelle, J, Paraschiv, F \& Fichtner, W (2016), 'Extended forecast methods for day-ahead electricity spot prices applying artificial neural networks,' Applied Energy, 162, pp. 218-230.

Kiesel, R \& Paraschiv, F (2017), 'Econometric analysis of 15-minute intraday electricity prices,' Energy Economics, 64, pp. 77-90.

Koenker, RW (2005), Quantile Regression, Cambridge University Press.

Lütkepohl, H (2005), New Introduction to Multiple Time Series Analysis, Springer-Verlag, Berlin.

Maciejowska, K \& Nowotarski, J (2016), 'A hybrid model for GEFCom2014 probabilistic electricity price forecasting,' International Journal of Forecasting, 32(3), pp. 1051-1056. 
Maciejowska, K \& Weron, R (2016), 'Short- and mid-term forecasting of baseload electricity prices in the U.K.: The impact of intra-day price relationships and market fundamentals,' IEEE Transactions on Power Systems, 31(2), pp. 994-1005.

Marcjasz, G, Uniejewski, B \& Weron, R (2018), 'On the importance of the long-term seasonal component in day-ahead electricity price forecasting with NARX neural networks,' International Journal of Forecasting, dOI: 10.1016/j.ijforecast.2017.11.009.

Misiorek, A, Trück, S \& Weron, R (2006), 'Point and interval forecasting of spot electricity prices: Linear vs. non-linear time series models,' Studies in Nonlinear Dynamics \& Econometrics, 10(3), p. Article 2.

Nowotarski, J, Tomczyk, J \& Weron, R (2013), 'Robust estimation and forecasting of the long-term seasonal component of electricity spot prices,' Energy Economics, 39, pp. 13-27.

Nowotarski, J \& Weron, R (2015), 'Computing electricity spot price prediction intervals using quantile regression and forecast averaging,' Computational Statistics, 30(3), pp. 791-803.

Nowotarski, J \& Weron, R (2018), 'Recent advances in electricity price forecasting: A review of probabilistic forecasting,' Renewable and Sustainable Energy Reviews, 81, pp. 1548-1568.

Pinson, P (2013), 'Wind energy: Forecasting challenges for its operational management,' Statistical Science, 28(4), pp. 564-585.

Rintamäki, T, Siddiqui, AS \& Salo, A (2017), 'Does renewable energy generation decrease the volatility of electricity prices? An analysis of Denmark and Germany,' Energy Economics, 62, pp. 270-282.

Schmidhuber, J (2015), ‘Deep learning in neural networks: An overview,' Neural Networks, 61, pp. 85-117.

Steinert, R \& Ziel, F (2019), 'Short-to mid-term day-ahead electricity price forecasting using futures,' The Energy Journal, 40, pp. 105-127.

Uniejewski, B, Nowotarski, J \& Weron, R (2016), 'Automated variable selection and shrinkage for day-ahead electricity price forecasting,' Energies, 9, p. 621.

Uniejewski, B \& Weron, R (2018), 'Efficient forecasting of electricity spot prices with expert and LASSO models,' Energies, 11, p. 2039.

Uniejewski, B, Weron, R \& Ziel, F (2018), 'Variance stabilizing transformations for electricity spot price forecasting,' IEEE Transactions on Power Systems, 33(2), pp. 2219-2229.

Weron, R (2014), 'Electricity price forecasting: A review of the state-of-the-art with a look into the future,' International Journal of Forecasting, 30(4), pp. 1030-1081.

Ziel, F (2016), 'Forecasting electricity spot prices using LASSO: On capturing the autoregressive intraday structure,' IEEE Transactions on Power Systems, 31(6), pp. 4977-4987.

Ziel, F \& Steinert, R (2018), 'Probabilistic mid- and long-term electricity price forecasting,' Renewable and Sustainable Energy Reviews, 94, pp. 251-266.

Ziel, F \& Weron, R (2018), 'Day-ahead electricity price forecasting with high-dimensional structures: Univariate vs. multivariate modeling frameworks,' Energy Economics, 70, pp. 396-420. 


\section{HSC Research Report Series 2019}

For a complete list please visit http://ideas.repec.org/s/wuu/wpaper.html

01 Electricity price forecasting by Katarzyna Maciejowska and Rafał Weron 